\title{
An Approach to Developing Adaptive Electronic Educational Course
}

\author{
R.Yu. Tsarev ${ }^{1}$, T.N. Yamskikh ${ }^{1}$, I.V. Evdokimov ${ }^{1}$, A.V. Prokopenko ${ }^{1}$, \\ K.A. Rutskaya ${ }^{1}$, V.N. Everstova ${ }^{2}$ and K.Yu. Zhigalov ${ }^{3,4}$ \\ ${ }^{1}$ Siberian Federal University, Krasnoyarsk, Russia \\ ${ }^{2}$ North-Eastern Federal University, Yakutsk, Russia \\ ${ }^{3}$ V.A. Trapeznikov Institute of Control Sciences of Russian Academy of Sciences, Moscow, \\ Russia \\ ${ }^{4}$ Moscow Technological Institute, Moscow, Russia \\ tsarev.sfu@mail.ru
}

\begin{abstract}
Modern information and telecommunication technologies allow not only to provide information required for training students in electronic form, they promote changes in educational process. The electronic information educational environment provides the necessary mechanism for implementing student's individual trajectories of training. Depending on his or her learning progress the process can vary from basic to intensive, with changes in volumes of information provided. One of the approaches to developing an adaptive electronic educational course is described in this paper. The tree of concepts mastered by students is considered as a basis for knowledge component organization. The tree of concepts will allow not only to organize theoretical material of the course in the form of the separate blocks of educational information intended for studying but also to determine a necessary and sufficient set of questions for testing students.
\end{abstract}

Keywords: Electronic Educational Course, Individual Trajectory, Tree of Concepts, Thesaurus.

\section{Introduction}

Application of information technologies in education allows to expand the range of educational technologies, to use new methods and ways to organize educational process. It is necessary to understand information technologies more widely, than direct representation of information required for training students in electronic form. Today a number of electronic information educational environments and platforms have been created. They allow to place educational and methodical materials within an electronic course, and transform presentation of the material and assessment results.

The purpose of electronic information educational environments and platforms application consists in providing interactive access to information educational resources of the course: theoretical materials, practical and independent tasks, tests, methodical instructions for working with the course and its separate elements. As a rule, electron- 
ic educational courses are located on the higher education institution website. However, educational and methodical materials representing intellectual property are in the access closed for external users, and this access can be provided only to the authorized users of the system $[1,2]$.

In the course of work in the electronic information educational environment of higher education institution students have an opportunity to use various training courses, information resources, including multimedia components of training materials, they can independently master training material in convenient time and in necessary amount, take tasks and tests in a desirable order [3]. Regular and up-to-date information provision on assessment of tasks and tests taken by students is the advantage of electronic educational courses [4]. These courses provide a student with an opportunity to interact with the teacher and the other students out of classroom by means of interactive forms of an electronic educational course [5]. Implementation of interactive forms of education promotes intensification of training process, allow to activate and increase the efficiency of student independent work. Moreover, application of electronic tutorials creates students responsibility, self-motivation and independence [6].

Nowadays one of the actual directions in education development is organization of such training process which considers individual capabilities and needs of the trainee as the subject of educational process. Information and telecommunication technologies and electronic management systems in education make forming of individual trajectories of the course studying possible and reasonable.

The principles of one of the approaches to developing an adaptive electronic educational course where adaptation is realized by means of student's trajectory in the course depending on his or her current assessments are described in this article.

\section{Form and Content of the Adaptive Electronic Educational Course Section}

\subsection{General Scheme of an Adaptive Electronic Educational Course Functioning}

To consider the procedures allowing to create the adaptive electronic educational course (AEEC), we will start with the essence of adaptive information and telecommunication technologies in education.

To understand what AEEC should do, it is necessary to provide a situation which arises in the course of studying the discipline, and that lack of necessary knowledge and practical experience faced by the student in the course of learning. Having provided a task mentally, it is necessary to choose decision strategy and to determine necessary resources.

Most often at this stage a student asks himself the following questions: What should I do? How should I do it? How do I know whether I do it correctly?

To answer these questions, it is necessary to contact with students (teacher and/or AEEC): to show, to instruct, to give advice, etc. 
Table 1 contains standard questions arising in the course of performing new or difficult tasks in the block of training material, and answers to them.

Table 1. Questions arising in the course of working with AEEC.

\begin{tabular}{ll}
\hline $\begin{array}{l}\text { Questions or requirements of } \\
\text { the student }\end{array}$ & \multicolumn{1}{c}{ AEEC Answers } \\
\hline What should I do it for? & Explanations, example, consequences \\
What is it? & $\begin{array}{l}\text { Definitions, illustrations, descriptions } \\
\text { What refers to it? }\end{array}$ \\
How I do it? & $\begin{array}{l}\text { Procedure, interactive reference books, ready methods } \\
\text { (flowcharts, algorithms, consultations) }\end{array}$ \\
How and why did it happen? & Explanation, example or demonstration \\
Give me an example... & Examples \\
Teach me... & Interactive training, practical activities with feedback \\
Help me... & Interactive consultations \\
Advise me... & Ready methods, flowcharts, algorithms, consultations \\
Let me try... & Practical work \\
Supervise me & Tracking systems \\
Estimate me & Assessments or tests \\
Understand me & Feedback with the report, reasoning, interpretation; the tracking \\
& systems monitoring actions of the user or communication \\
How does it work? & Explanations and examples \\
Compare this and that for me & Comparative explanations and descriptions \\
Predict for me & Descriptions and demonstration of consequences \\
Where am I? & Management systems, tracking systems, communication types \\
What's next? & (You are here) \\
\hline
\end{tabular}

The table is not limited with the listed points, but allows to create a base for creative thinking in AEEC planning. It is necessary to remember that the number of questions is limited, and all related information can be determined precisely. Each trainee can use more convenient and understandable questions-answers connections, realizing thereby individual training. It is clear, that individual classes with a student (teacher/AEEC) is the most perspective educational technique.

The sequence of material presentation, depth and knowledge of information will individually vary for the specific student within an educational trajectory. When one of educational methods does not work, the student will try to choose another or concretizes his request to AEEC. The teacher can choose various help strategies. Besides, he can adjust the student's work, approving the strategy chosen or offering another one, more convenient for the achievement of an effective objective. It also helps to implement one of the promising pedagogical methods - model of mastering levels regarding cultural action pattern [7]. 
So, the offered approach to developing AEEC is based not only on educational concepts but also on educational resources and objectives. Resource and objective approach to developing information systems in education is not new. However there are not enough effective, simple and easily implemented in practice methods of their development. Besides, the existing IT decisions do not contain the means of choosing student's individual trajectories of training, depending on his or her progress, i.e. means of AEEC. The approach offered is especially intended for the solution of these problems.

Procedures of this technology are guided by a tree of concepts that allows not only to organize a theoretical course material in the form of the separate blocks of educational information intended for learning but also to determine a necessary and sufficient set of questions for testing students in the course of studying, or a set of competences (competence-based approach in education). Thus, all procedures can be divided into three levels:

1. The top level, based on a tree of objectives, provides preliminary determination of an educational trajectory option.

2. The middle level is guided either by a tree of concepts, or by a set of competences, or by both simultaneously. The task of this level consists in determination of a final decision.

3. The lower level is intended for ensuring execution of educational methods: intellectual information support of students.

The interrelation between procedures of the offered approach to developing AEEC is provided in Fig. 1.

The general scheme of AEEC functioning allowing to trace the sequence of system blocks inclusion in operation is shown in Fig. 2.

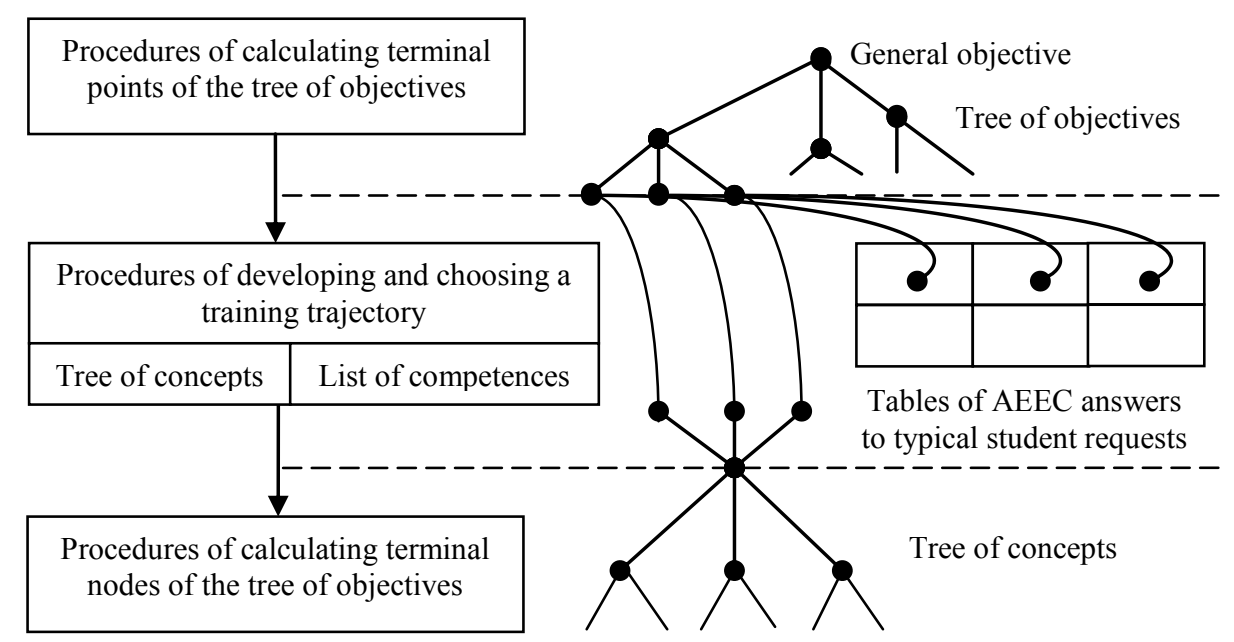

Fig. 1. Interrelation of levels in procedures of the offered approach to developing AEEC. 


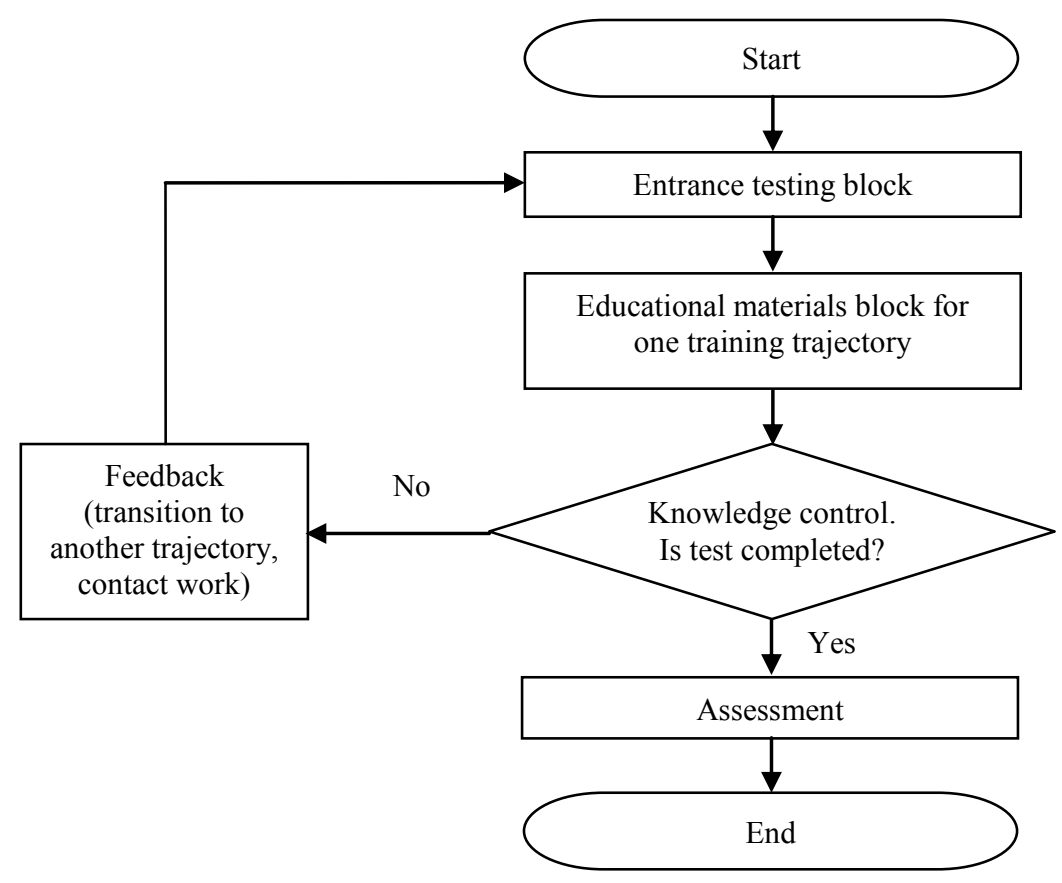

Fig. 2. General scheme of AEEC functioning.

In Fig. 2 the block of entrance testing is based on expanded psychological testing of students with control of residual knowledge in the competences of the disciplines preceding studying this specified curricular course. The results of such testing are subsequently used to form conclusions about a trajectory of training in the block of training material.

\subsection{Tree of Concepts as Formal Representation of Theoretical Material of an Electronic Course}

Developing an adaptive electronic educational course assumes first of all the organization of theoretical material representing knowledge component. "Knowledge is in turn determined as a form of existence and systematization of the person cognitive activity results or as a form of social and individual memory, the result of an object structuring and reasoning in the course of learning" [8].

When studying theoretical material, connectivity of the provided information, integrity of perception is reached only by means of concepts [9]. Thus, to describe any knowledge we need a necessary condition - the developed conceptual framework.

This idea is proved by N.I. Kondakov, claiming that the concept is a complete set of judgments, that is thoughts in which something is affirmed about distinctive signs of the object researched, which kernel are judgments about the most general and at the same time essential signs of this object, while knowledge - complete and systema- 
tized set of scientific concepts about regularities of the nature, society and thinking, stored by mankind in the course of vigorous reformative productive activity and directed to further knowledge and changes of the objective world [10].

The organization of theoretical material within an adaptive electronic educational course is carried out on the basis of a tree of concepts. A root of a tree is the most general concept, during mastering the course this concept reveals and disaggregated that is formally presented in the form of tree branches.

The set of concepts of one level having the general ancestor represent structural model of this concept ancestor and completely determine it in total. Each concept provided in a tree is also described by the phenomenological model allocating it from a set of similar concepts, the definition of the concept itself is provided in a separate component of the course - the thesaurus reflecting interrelation between concepts.

Studying of the course can be formally presented in the form of a bypass of a tree of concepts in depth. The advantage of the approach to organizing theoretical course material on the basis of a tree of concepts is the possibility to allocate separate logically connected blocks of educational information including one or several nodes of a tree of concepts depending on the concept complexity. The concept structural model allows to create the bank of the test questions necessary and sufficient to control knowledge acquired by students.

So, we have come to a conclusion that theoretical material within AEEC is carried out on the basis of a tree of concepts which, in turn is nothing else than a kind of thesauruses (dictionaries). To organize information search and to maintain such thesaurus the descriptor classification system of information (still called by descriptive) often is effectively used as its language approaches a natural language of the description of information objects.

The majority of thesauruses standards versions specify connection of terms with concepts of subject domain. The ISO standard (ISO 5963:1985) underlines that the indexing term - is representation of concept preferably in the form of a noun or a noun phrase [11]. At the same time the concept is considered as a unit of thought which is created mentally to reflect all or some properties of specific or abstract, reallife or mental object. Concepts exist as abstract entities, irrespective of terms which express them.

Thus, thesauruses developers assume that the concept of subject domain usually has several possible options of lexical representation in the text which are considered as synonyms. The descriptor is chosen among such synonyms- the term which is considered as the main method of reference to concept within the thesaurus.

The descriptor method of classification in our case consists in the following.

First, a set of the keywords or phrases describing subject domain of AEEC or a set of uniform objects is selected; at the same time there can be synonyms among keywords. To form such set of keywords or phrases three main sources of keywords or phrases selection for a tree of concepts are used.

The first source - professional and common cultural competences defined by Federal state educational standard of higher education bound to a lecture subject provided by the author of the course in the working program of the discipline (in the curriculum of a profile of preparation). 
The second source - a subject of specific lecture from the working program of the discipline and other materials relating to this subject.

The third source - phenomenological model as a set of knowledge determining interrelation between various observations of the phenomena (phenomena) according to the fundamental theory, but which do not follow from this theory directly. The phenomenological model transform request like "formulation of a problem" by means of modeling the content of a request situation with thesaurus concepts into Boolean expression "conjunction of disjunctions" over the concepts of the thesaurus:

$$
\bigcap_{i} c_{i j}
$$

where $c_{i j}$ - thesaurus concepts.

Elements of disjunction can be concepts of the thesaurus which are considered as similar in their meaning - they are connected among themselves by thesaurus ways of a certain type.

Secondly, the chosen keywords and phrases are exposed to normalization, i.e. one or several most common are taken from a set of synonyms - descriptors. Other terms from a synonymic row included in the thesaurus are called askriptor or nondescriptors. They are used as the auxiliary elements, text entrances helping to find suitable descriptors. The descriptors are available in both brief and verbose formats.

The set of descriptors should meet the following requirements:

- the opportunity to describe the subject of the majority of texts in subject domain should be provided by means of the descriptors allocated;

- to reduce subjectivity of indexing the set of descriptors should not include sets of close descriptors; for this purpose classes of relative equivalence, when sets of close, but different concepts are reduced to one descriptor are created;

- the descriptor should be formulated unambiguously, its value implied within the thesaurus should be clear to the user. If it is not possible to find the unambiguous and clear descriptor, the term taken as a descriptor is supplied with the comment.

Such substantial types of relations between descriptors are most often not reflected in the detailed list of the thesaurus relations, and are registered by means of a small set of relations which are usually divided into two types: hierarchical and associative.

Many guidebooks and standards emphasize that the hierarchical relations in the thesaurus should be established when the relations are true irrespective of a context only in such cases descriptors of the thesaurus can be organized in hierarchy. This recommendation is connected with the fact that in information search it is usually very difficult to determine accurately a context of the term usage and to understand whether this or that relation is applicable in this context.

So, for the software it is possible to specify that it is a software product as it is the internal characteristic of the software as the result of human activities exposed in the market of the mass buyer as goods. At the same time it is wrong to specify that the software is application program packages as there are, for example, operating systems which are not application-oriented.

The basic purpose of establishing associative relations between thesaurus descriptors - specifying on additional descriptors, useful for indexing or search. The 
relation of association is associative and not hierarchical. It is difficult to define the associative relation. All types of the relations, except a synonymy and the relation "sort - type" are allowed to be included in the associative relation.

Thirdly, the dictionary of descriptors, i.e. the dictionary of the keywords and phrases selected as a result of normalization procedure is created.

\subsection{Tree of Concepts as Formal Representation of Theoretical Material of an Electronic Course}

Developing individual training trajectory represents the multidimensional process aimed at providing the trainee independence and initiative, possibility to use his or her personal and cognitive potential and professional growth within educational process. For example designing different educational situations for bachelor students need specific educational information units to initiate students' reflection on content assimilation [12]. This kind of reflection being implemented in an activity mode could help students to develop themselves as professionals consciously and purposefully.

The process of individual training trajectory formation assumes building a sequence of educational information units within a certain course which mastering will provide a trainee with a chance to achieve educational goal and to obtain a set of the competences assumed by the curriculum and the working program of the discipline.

Separation of logically complete information blocks to study theoretical material allows to create different trajectories taking into account personal abilities and progress of the student. It was offered to select three trajectories relatively corresponding to studying the material with estimation marks "satisfactory", "good", "excellent".

The content of each educational information block is fixed, however, the form of material representation for each individual trajectory of training differs. For the students studying the discipline following a trajectory "excellent", information is presented in a more squeezed form, assuming that this category of students possesses ability to acquire theoretical material without its detailed explanation. On the contrary, for the students following a trajectory "good" the material is presented in details, with examples and illustrations.

The volume of each block of educational information, and, therefore, the corresponding block of test questions should not be large. It is offered to use minimum possible set of interconnected concepts determined by a tree of concepts for this discipline.

Separate blocks of educational information are formed on the basis of a set of the interconnected concepts, terms, keywords which define precisely the elementary block of theoretical material provided to students during their work with an electronic educational course. Within a tree of concepts, blocks of educational information represent one or several interconnected tree nodes. The concepts presented in a tree are given in the glossary of an electronic educational course.

The most important element of the electronic information educational environment is to control student knowledge throughout the trajectory of his learning [13]. Proceeding from this situation, the student takes the test defining his further training trajectory after studying each block of educational information. Besides assessing assim- 
ilation of theoretical knowledge blocks of test questions have to be directed to support student motivation and stimulate interest in further studying of the discipline.

In the beginning of studying the discipline with electronic educational course the student takes entrance test which defines his initial trajectory within a course. As a rule, entrance testing estimates residual knowledge of the disciplines, previous in structure of educational program.

If the test shows that a student hasn't satisfied the condition sufficient for continuing the course there is a kickback, and a student is forced to study repeatedly the same block of theoretical information and to take the test once again (see Fig. 3).



Fig. 3. Developing individual training trajectory with categories of estimation marks satisfactory-good-excellent with kickbacks.

In case of additional studying the block of educational information represented in different form, as a rule, from the lower trajectory is offered to the student. If a kickback has happened after taking the trajectory "good", it is possible to execute return on higher trajectory, believing that the new representation of the same information taking into account the material studied will be more available to the student.

Contact work with the teacher for studying this block of theoretical information is required in case of repeated negative test result.

\section{Conclusion}

The result of applying the above described approach to developing an adaptive electronic educational course is formalization of the basic principles, methods and technology of implementing adaptive approach to studying the disciplines with individual 
educational trajectories taking into account the current level of mastering educational material by a certain student.

The created electronic educational course considers the student individual capabilities and on the basis of assessment automatically transfers it to higher or low trajectory. Thus we see the adaptive nature of taking the course and studying theoretical material.

Application of this approach is directed to decrease the classroom loading connected with transferring lectures to student independent work when all theoretical material is provided in appropriate units of an adaptive electronic educational course. It will encourage activity and independence of students that is important not only for studying a separate course, but also for training students as future specialists capable to solve problems independently.

\section{References}

1. Elliott, D., Hopfgartner, F., Leelanupab, T., Moshfeghi, Y., Jose, J.M.: An architecture for life-long user modelling. In: Lifelong User Modelling Workshop held in conjunction with User Modeling, Adaptation and Personalisation, pp. 1-8. Torento, Italy (2009).

2. Nguyen, L., Do, Ph.: Learner Model in Adaptive Learning, world academy of science. Engineering and Technology 45, 395-400 (2008).

3. Li, Q., Zhong, S., Wang, P., Guo, X., Quan, X.: Learner model in adaptive learning system. Journal of Information and Computational Science 7(5), 1137-1145 (2010).

4. You, D., Shen, L., Peng, S., Liu, J.: Flexible collaborative learning model in e-learning with personalized teaching materials. Advances in Intelligent and Soft Computing 105, 127-131 (2011).

5. Jia, B., Yang, Y., Zhang, J.: Study on learner modeling in adaptive learning system. Journal of Computers (Finland) 7, 2585-2592 (2012).

6. Jia, B., Yang, Y., Zhang, J.: Representation and acquisition of feature value of learner model in adaptive learning system. Advances in Intelligent and Soft Computing 122, 371377 (2011).

7. Bazhenova, K., Znamenskaya O.: Teacher's beliefs as a factor of individual progress of pupil. In.: European Proceedings of Social \& Behavioural Sciences EpSBS, pp. 100-108. Kazan, Russia (2017).

8. Hsu, W.-C., Li, C.-H.: A competency-based guided-learning algorithm applied on adaptively guiding e-learning. Interactive Learning Environments 23(1), 106-125 (2015).

9. Op Den Akker, R., Hofs, D., Hondorp, H., Op Den Akker, H., Zwiers, J., Nijholt, A.: Supporting engagement and floor control in hybrid meetings. Lecture Notes in Computer Science (including subseries Lecture Notes in Artificial Intelligence and Lecture Notes in Bioinformatics) 5641 LNAI 276-290. (2009).

10. Kondakov, N.I.: Logicheskij slovar' [Logical dictionary]. Science, Moscow, Russia (1971).

11. ISO 5963:1985 Documentation. Methods of documents analysis, determinations of their subject and selection of indexing terms.

12. Bazhenova, K.A.: Designing educational situations for bachelor students in Pedagogics Moscow University Bulletin 3, pp. 73-83, (2014).

13. Rus, V., D'Mello, S., Hu, X., Graesser, A.C.: Recent advances in conversational intelligent tutoring systems. AI Magazine 34(3), 42-54 (2013). 